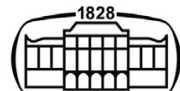

AKADÉMIAI KIADÓ

Pollack Periodica •

An International Journal

for Engineering and Information Sciences

16 (2021) 2, 80-85

DOI:

$10.1556 / 606.2021 .00307$

(c) 2021 The Author(s)

\title{
Optimization of drilling performance using various metaheuristics
}

\section{Shaymaa Alsamia ${ }^{1}$, Dhorgham S. Ibrahim ${ }^{2 *} \odot$ and Hazim N. Ghafil ${ }^{3,4}$}

${ }^{1}$ Department of Structures and Water Resources, Faculty of Engineering, University of Kufa, Kufa, Iraq

${ }^{2}$ Petroleum and Natural Gas Institute, Faculty of Earth Science and Engineering, University of Miskolc, 3515, Egyetemváros, Miskolc, Hungary

${ }^{3}$ Department of Mechanical Engineering, Faculty of Engineering, University of Kufa, Kufa, Iraq

${ }^{4}$ Institute of Energy Engineering and Chemical Machinery, Faculty of Mechanical Engineering and Informatics, University of Miskolc, 3515, Egyetemváros, Miskolc, Hungary

Received: November 29, 2020 • Revised manuscript received: January 17, 2021 • Accepted: March 3, 2021

Published online: April 27, 2021

\section{ABSTRACT}

The most crucial function in drilling wells is the rate of penetration, which is modeled by many researchers, and the best one is Young-Bourgyen model, which is used in this study. Eight factors affecting rate of penetration have been studied and approved in developing a mathematical equation that shows the combined effects of these variables on rate of penetration optimization. This paper presents an efficient way to find the optimum values for parameters of the Young-Bourgyen model using metaheuristic algorithms. An actual drilling data was used from Khangiran field to calculate the difference between the actual penetration rate and the predicted one by different optimization algorithms. Particle swarm optimization, dynamic differential annealing optimization, artificial bee colony, gray wolf optimization, Harris hawk's optimization, flower pollination algorithm, firefly algorithm, whale optimization algorithm, and sine cosine algorithm are used to find best possible solution.

\section{KEYWORDS}

optimization algorithms, formation rocks, penetration rate, drilling performance, dynamic differential annealed optimization, swarm intelligence

\section{INTRODUCTION}

The demand for underground resources, like minerals, groundwater in aquifers, and groundsource energy, has increased dramatically in recent decades. Drilling is required for any of these resources to be extracted [1]. But, like every other instrument, the drill, has a range of problems that are not well discussed and more detail needs to be given. Optimized drilling is the system or the program that can be used to reduce the cost of the deep water, oil or gas well for the operator to the minimum [2]. The selection of drilling parameters (drilling optimization), lead to the optimum prediction of the drilling rate, which is critical to minimize the cost of drilling per foot [3]. Drilling a hole in the ground to find water in an aquifer or extracting oil and gas is a complex and multifaceted activity that is subject to substantial sources of variability while the physics of drilling is the same worldwide. Geological conditions, contractor expertise, availability of equipment, well specification, and various other factors will contribute to a wide range in drilling performance [4]. Drilling optimization aims to improve controllable variables like weight on bit and bit rotation speed during the drilling process to achieve optimum drilling rate [5]. Optimization of costs is a procedure, and its main aim is to minimize the cost by setting the intervention parameters to optimum level. Main drilling variables considered to have an impact on the penetration rate of drilling are not well known and difficult to model. There are several recommended 
mathematical models that have attempted to incorporate known drilling parameter relations. The proposed models worked to optimize the process of drilling by choosing the best rotational speed and weight to achieve the lowest cost [6]. Scientists have attempted to suggest some clarified models to create a relation between the drilling rate and its major variables. Well drilling is a multivariable mathematical issue in which the Rate Of Penetration (ROP) depends on controllable drilling variables. Wettability and capillary rise are of essential importance to drilling fluid formulation [7].

The most crucial function in drilling wells is the rate of penetration which is modeled by many researchers, and one of the best is Young-Bourgoyne model, which is used in this study [8]. This paper presents an efficient way to find the optimum values for parameters of the Young-Bourgoyne model using metaheuristic algorithms. An actual drilling data was used from Khangiran field to calculate the difference between the actual penetration rate and the predicted one by Particle Swarm Optimization (PSO) [9], Dynamic Differential Annealing Optimization, (DDAO) [10], Artificial Bee Colony (ABC) [11], Grey Wolf Optimization (GWO) [12], Harris Hawks Optimization (HHO) [13], Flower Pollination Algorithm (FPA), Firefly Algorithm (FF), Whale Optimization Algorithm (WOA), and Sine Cosine Algorithm (SCA). The results from these metaheuristics are compared and discussed, and no one of them could find acceptable solution. Therefore, a unique procedure has been followed to introduce a robust mathematical function can describe ROP precisely. Also, the predicted penetration rate from the proposed procedure was compared with a previous work had used genetic algorithm (GA) to find ROP.

\section{METAHEURISTICS}

Metaheuristics are powerful mechanism to search for best possible solutions among many other solutions available. They are optimization algorithms that they differ in their efficiency, convergence speed, complexity, etc. The most important issue in the efficiency term is the capability of the algorithm to escape from local minimum values in the search space of the optimization problem. All the optimization algorithms in this study follow the same principle to search for the global minimum, which is trying random solution many times until they reach a suitable solution. In brief, in order to determine the optimum values of $\mathrm{Y}-\mathrm{B}$ model coefficients, the metaheuristics follows the flow chart presented in Fig. 1.

\section{RATE OF PENETRATION}

The rate of penetration, is the speed of breaks the rock under the drill bit to deepen the borehole [14]. It is normally measured in feet per minute or meters per hour.

ROP is pointed from the field and research experts and depends on several variables like properties of rock

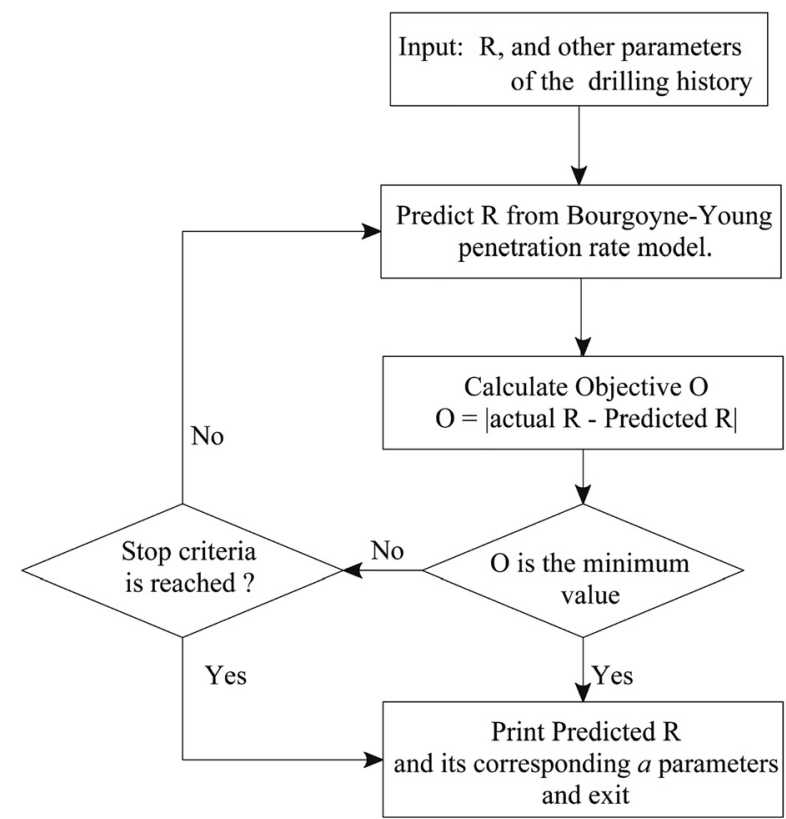

Fig. 1. Flow chart representing the drilling optimization process

formation, bit type and size, weight on bit, rotation speed, rheology of drilling fluid, hydraulic system, and depth of the formation. Eight factors affecting ROP have been studied and approved in developing a mathematical equation that shows the combined effects of these variables on ROP optimization. Young-Bourgoyen model and the functional relations in this equation are as follow in Eqs. (1)-(9):

$$
\begin{gathered}
R=f_{1} \times f_{2} \times f_{3} \times f_{4} \times f_{5} \times f_{6} \times f_{7} \times f_{8}, \\
f_{1}=e^{2.303 a_{1}}, \\
f_{2}=e^{2.303 a_{2}(10000-D),} \\
f_{3}=e^{2.303 a_{3} D^{0.69}\left(g_{p}-9\right),} \\
f_{4}=e^{2.303 a_{4} D\left(g_{p}-\rho_{c}\right),} \\
f_{5}=\left[\begin{array}{l}
\frac{W}{d_{b}}-\left(\frac{W}{d_{b}}\right)_{t} \\
4-\left(\frac{W}{d_{b}}\right)_{t}
\end{array}\right]^{a_{5}}, \\
f_{6}=\left(\frac{N}{60}\right)^{a_{6}}, \\
f_{7}=e^{-a_{7} h}, \\
f_{8}=\left(\frac{F_{j}}{1000}\right)^{a_{8}},
\end{gathered}
$$

where $a_{1}$ to $a_{8}$ are constants; $D$ is the true vertical depth [m]; $d_{b}$ is the bit diameter $[\mathrm{cm}] ; F_{j}$ is the jet impact force, $[\mathrm{N}] ; g_{p}$ is the pore pressure gradient, $\left[\mathrm{N} / \mathrm{m}^{2}\right] ; h$ is the fractional bit tooth wear [\%]; $\rho_{c}$ is the equivalent circulating density, $\left[\mathrm{kg} / \mathrm{m}^{3}\right] ; N$ is the rotary speed, $[\mathrm{rpm}] ; R$ is the rate of 
Table 1. Drilling history from field

\begin{tabular}{lcccccccrr}
\hline Well No. & $R(\mathrm{~m} / \mathrm{h})$ & $D(\mathrm{~m})$ & $W(\mathrm{~N})$ & $d_{b}(\mathrm{~cm})$ & $N(\mathrm{rpm})$ & $\rho_{c}\left(\mathrm{~kg} / \mathrm{m}^{3}\right)$ & $h(\%)$ & $g_{b}\left(\mathrm{~kg} / \mathrm{m}^{3}\right)$ & $F_{j}(\mathrm{~N})$ \\
\hline Well 50 & 15.43 & 107.93 & 3.40 & 66.04 & 130 & $1,056.90$ & 0.25 & 896.3 & 435.45 \\
Well 50 & 12.65 & 430.18 & 6.80 & 44.45 & 130 & $1,193.50$ & 0.25 & $1,032.9$ & 805.59 \\
Well 47 & 7.41 & 109.45 & 6.80 & 66.04 & 130 & $1,060.49$ & 0.25 & 913.10 & 730.74 \\
Well 47 & 4.54 & 463.11 & 4.54 & 44.45 & 110 & $1,222.26$ & 0.38 & $1,056.9$ & 962.99 \\
Well 46 & 2.23 & 540.24 & 3.40 & 44.45 & 110 & $1,234.24$ & 0.25 & $1,072.4$ & 537.51 \\
Well 42 & 2.90 & 600.30 & 4.54 & 44.45 & 110 & $1,294.16$ & 0.5 & $1,137.1$ & 600.56 \\
Well 39 & 1.74 & 579.27 & 4.08 & 44.45 & 100 & $1,258.21$ & 0.5 & $1,096.4$ & 537.96 \\
Well 29 & 7.90 & 480.18 & 6.80 & 44.45 & 90 & $1,246.23$ & 0.38 & $1,089.2$ & 996.10 \\
\hline
\end{tabular}

penetratin, $[\mathrm{m} / \mathrm{h}] ; W$ is the weight on bit, $[1,000 \mathrm{~N}] ;\left(W / d_{b}\right)$ is the threshold bit weight per meter of bit diameter at which the bit begins to drill, $[1,000 \mathrm{~N}]$.

There are eight unknown parameters in this model, which are dependent to the ground formation types. These eight parameters can be determined using previous drilling experiences. The function $f_{1}$ represents the effect of formation strength on penetration rate. The functions $f_{2}$ and $f_{3}$ show the effect of formation compaction on penetration rate. The function $f_{4}$ models the effect of overbalance across the whole bottom on penetration rate. The functions $f_{5}$ model the effect of bit weight and bit diameter on penetration rate. The functions $f_{6}$ model the effect of rotary speed on penetration rate. The function $f_{7}$ models the effect of tooth wear and, the function $f_{8}$ models the effect of bit hydraulics on penetration rate. The constants $a_{1}$ to $a_{8}$ are dependent on local drilling conditions and must be computed for each formation using the previous drilling data obtained in the area when detailed drilling data are available. In fact, the accuracy of this model is dependent to the coefficient values and therefore, applying a reliable mathematical technique to compute these constants. Bourgoyne and Young recommended multiple regression method to determine unknown coefficients. However, applying multiple regression method leads to physically meaningless values in some situations.

\section{STATISTICAL RESULTS}

Experimental data from Khangiran field [15] was used to show the efficiency of the proposed optimization procedure. Table 1 illustrates the drilling parameters for eight wells while Fig. 2 presents the predicated model using genetic algorithm in case of the first well (well 50). The solution of the regression problem in Fig. 2 is very far from the actual data, and this solution is provided in previous work using genetic algorithm [15]. It clear from the figure that this solution cannot be reliable to express the drilling model. The goal in this section is find better solution to fit the experimental data from drilling history in an acceptable formulation.

For the sake of finding better solution, nine optimization algorithms shown in Table 2 are employed. Also, a comparison among the results of these nine metaheuristics is made to discover which one is more efficient than the rest. Table 2 reveals the comparative statistical results of the nine algorithms used in this study. The run condition is 30 independent runs, 50 population sizes, 1,000 maximum number of iterations, and number of variables is 8 with range $0-1.5$. Most the algorithms return best solution with objective around 6.8 while the goal is reach zero objective value. It is obvious from Table 2 that no optimization algorithm can solve this regression problem perfectly [16]. All the competitive algorithms starts with random initial solution within the search space and this solution get improved during iterations. One trick is used to get better results than what is exists in Table 2 which is using combination of algorithms to solve the problem in this study. The procedure is to choose one optimization algorithm to solve the problem then its better solution will be the initial solution for the second algorithm. After improving the solution with the second algorithm, the best solution of the second algorithm will also be the initial solution for the third optimization algorithm and so on.

Thus, a combination of FF, ABC and GWO have been used to estimate a piecewise function that can formulates the 8th constants, which are expressed in the pies-wise function in Eq. (10). Figures 3 and 4 show the behavior of the function on the two periods. It is clear the predicted ROP matches exactly the real one from field and this is the best results can be found for this problem.

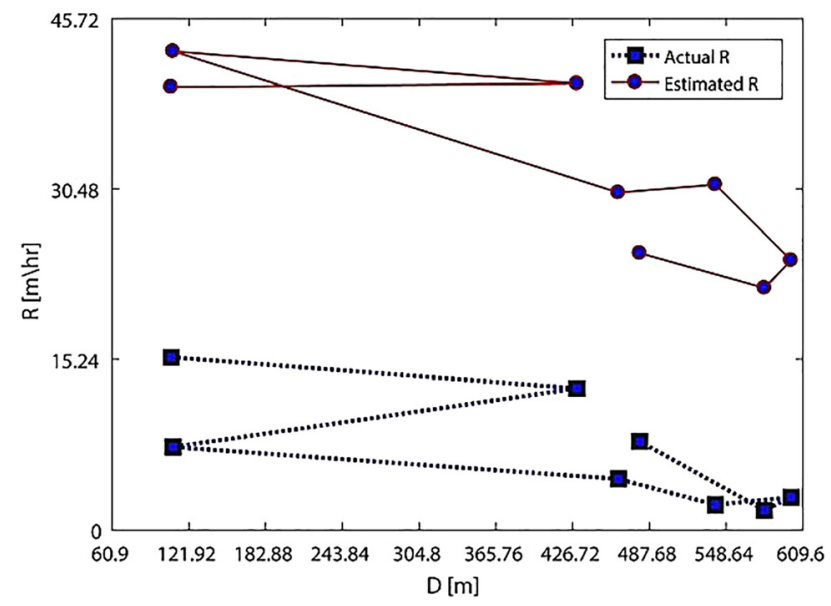

Fig. 2. The actual and calculated penetration rate using the genetic algorithm in Khangiran formation 
Table 2. Competitive results of the metaheuristics on Young-Bourgoyne model

\begin{tabular}{lcccc}
\hline Algorithm & Best & Worst & Mean & STD \\
\hline PSO & $6.9126 \mathrm{E}+01$ & $7.8203 \mathrm{E}+03$ & $1.1518 \mathrm{E}+03$ & $2.6607 \mathrm{E}+03$ \\
HHO & $6.9259 \mathrm{E}+01$ & $9.1215 \mathrm{E}+01$ & $7.2732 \mathrm{E}+01$ & $3.6638 \mathrm{E}+00$ \\
DDAO & $9.7440 \mathrm{E}+01$ & $1.7970 \mathrm{E}+02$ & $1.7014 \mathrm{E}+02$ & $1.9823 \mathrm{E}+01$ \\
ABC & $6.8457 \mathrm{E}+01$ & $7.5242 \mathrm{E}+01$ & $7.1984 \mathrm{E}+01$ & $3.0904 \mathrm{E}+00$ \\
GWO & $6.8466 \mathrm{E}+01$ & $1.0489 \mathrm{E}+02$ & $7.6302 \mathrm{E}+01$ & $8.5153 \mathrm{E}+00$ \\
FF & $6.8456 \mathrm{E}+01$ & $7.8203 \mathrm{E}+03$ & $5.7544 \mathrm{E}+03$ & $3.4844 \mathrm{E}+03$ \\
WOA & $6.9768 \mathrm{E}+01$ & $9.3706 \mathrm{E}+01$ & $7.9810 \mathrm{E}+01$ & $5.9615 \mathrm{E}+00$ \\
SCA & $7.5243 \mathrm{E}+01$ & $8.4012 \mathrm{E}+01$ & $7.7046 \mathrm{E}+01$ & $2.9858 \mathrm{E}+00$ \\
FPA & $6.8459 \mathrm{E}+01$ & $7.5243 \mathrm{E}+01$ & $7.2417 \mathrm{E}+01$ & $3.1305 \mathrm{E}+00$ \\
\hline
\end{tabular}

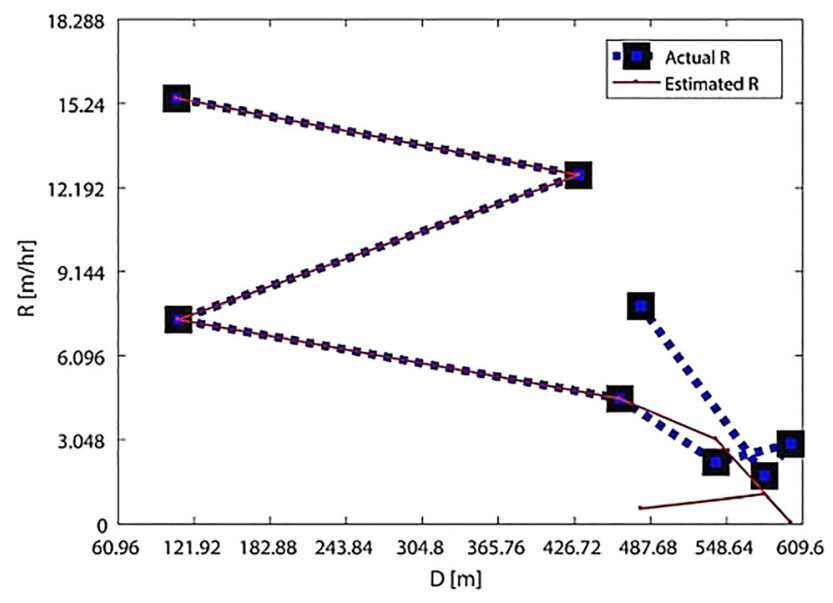

Fig. 3. The actual and calculated penetration rate using the combination of FF, $\mathrm{ABC}$ and GWO for depths equal or less than $457.2 \mathrm{~m}$ in Khangiran formation

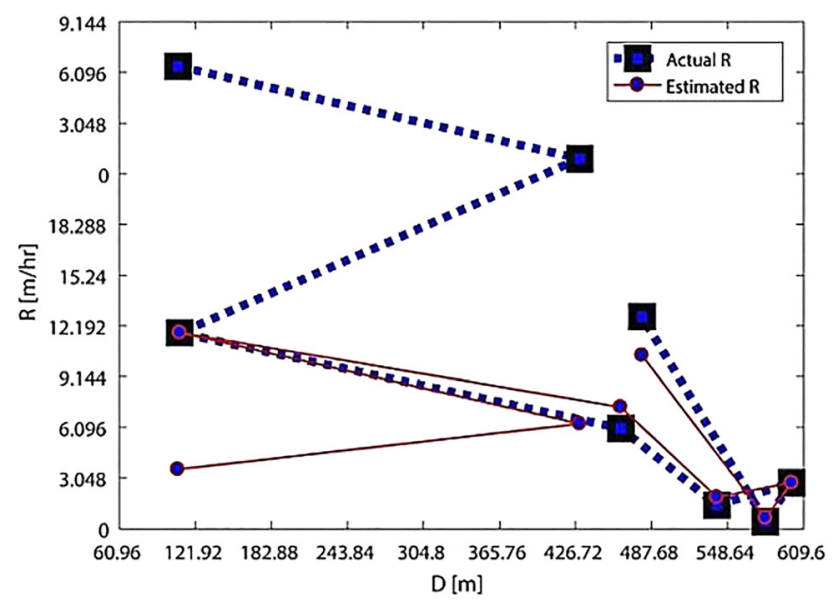

Fig. 4. The actual and calculated penetration rate using the combination of FF, $\mathrm{ABC}$ and GWO for depths greater than $457.2 \mathrm{~m}$ in Khangiran formation

$$
a_{i}= \begin{cases}A_{i} & D \leq 1500 \\ B_{i} & D>1500\end{cases}
$$

where $a_{i}$ defined as follows: $a_{1}=1.6133 \mathrm{E}+00$, $a_{2}=-2.2371 \mathrm{E}-04, a_{3}=-2.1120 \mathrm{E}-02, a_{4}=-3.6900 \mathrm{E}-04$, $a_{5}=1.7588 \mathrm{E}-01, a_{6}=2.5652 \mathrm{E}-03, a_{7}=-1.9975 \mathrm{E}+00$, $a_{8}=-7.4389 \mathrm{E}-01$, and $b_{i}$ defined as: $b_{1}=1.4995 \mathrm{E}+00$, $b_{2}=0.0000 \mathrm{E}+00, b_{3}=1.9875 \mathrm{E}-03, b_{4}=2.1926 \mathrm{E}-04$, $b_{5}=1.1886 \mathrm{E}-01, b_{6}=0.0000 \mathrm{E}+00, b_{7}=1.4811 \mathrm{E}+00$, $b_{8}=1.5000 \mathrm{E}+00$.

Figures 3 and 4 prove that for some engineering problems using one optimization algorithm is not enough and does not guarantee an acceptable solution. By using a combination of FF, ABC and GWO, more accurate solution was found, a solution more accurate than what is provided in previous work by [15]. Thus in this section, a comparison among nine optimization algorithm is made on drilling problem, novel procedure is introduced, and proved that the proposed procedure return better solution compared with previous work written for the same optimization problem.

\section{ANALYSIS FOR VARIOUS PARAMETERS}

There are eight unknown parameters in this model, which are dependent to the ground formation types. These eight parameters can be determined using previous drilling experiences. Also, some of these parameters are based on empirical correlations developed from laboratory studies. The previous solutions failed to provide a satisfactory and reliable tool for estimating penetration rate especially with increase the number of variables in the model. Optimization algorithms were used as an alternative approach in analysis of different parameters on rate of penetration and it show reasonably good results. Figure 5 and 6 presented the combination effect on ROP. As it can be seen, the optimum rate of penetration for the selected depth is obtained when $W=20,411.657-22,679.619 \mathrm{~kg}$, and $N=$ 125-130 rpm.

Although the rate of penetration depends mainly on the weight on bit and the speed of rotation, there are other factors such as the drilling fluid system, the hydraulic drilling system and the flow rate have a large impact if these variables are chosen carefully. The drilling model used in this study gave results identical to the field results if the values obtained through the optimization operations were selected and shown in Figs 5 and 6 with other variables being taken from the field data. The model also ensured that 


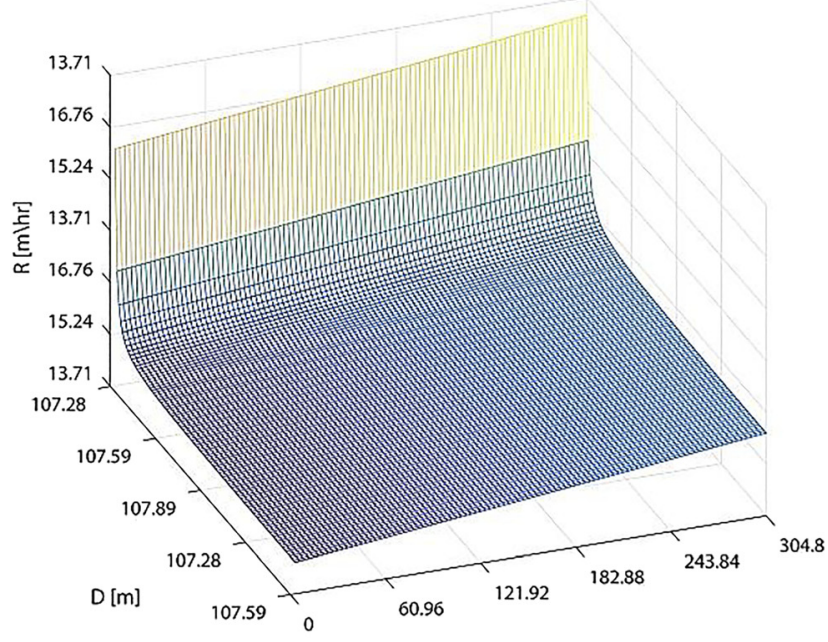

a)

Threshold bit weight per meter of bit diameter [N]

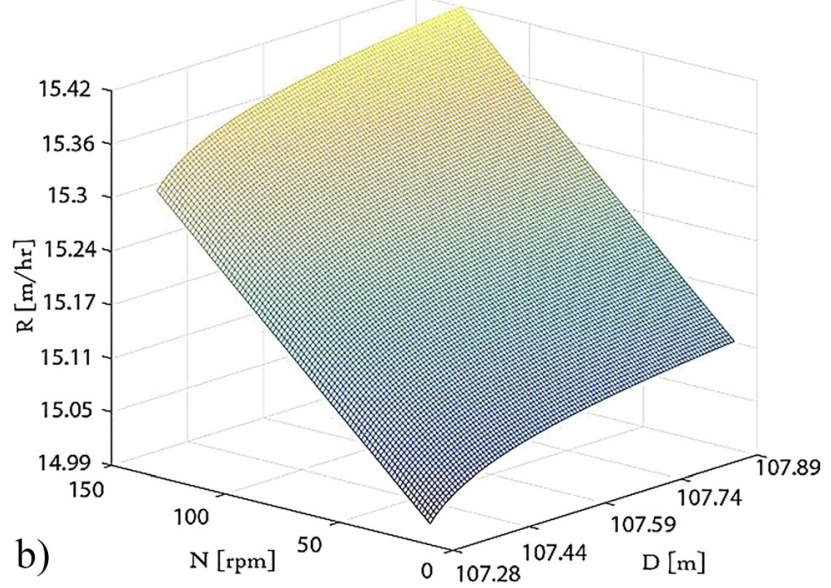

Fig. 5. a) Effect of $\left(W / d_{b}\right)$ and $D$ on ROP; b) effect of $N$ and $D$ on ROP

the values of the applied weight on bit and the rotational speed remained constant during the service of the bit or the period of operation of the drill bit during a fixed section of the rock layer. The model also takes into account the well deviation, the characteristics of the drilling fluid, the hydraulic drilling system and the movement of the drill string.

Drilling test is generally performed on a fixed layer and with various penetration rates, where Fig. 5 shows the relationship between rotation speed and penetration rate, while Fig. 6 represents the weights imposed on the bit. When the results of the penetration rate agree with each other, this means that the examination is acceptable, from the combined matrix of the rotation speed, the weight applied to the drill and the penetration rate of a section of rock drill, the optimization can be obtained, which can be seen in the figures below. It is represented by the shape of the surface of the penetration rate, the rotation speed and the weight applied to the drill bit, which is not bypassed, to prevent any failure and wear of the teeth of the drill bit or its bearings.
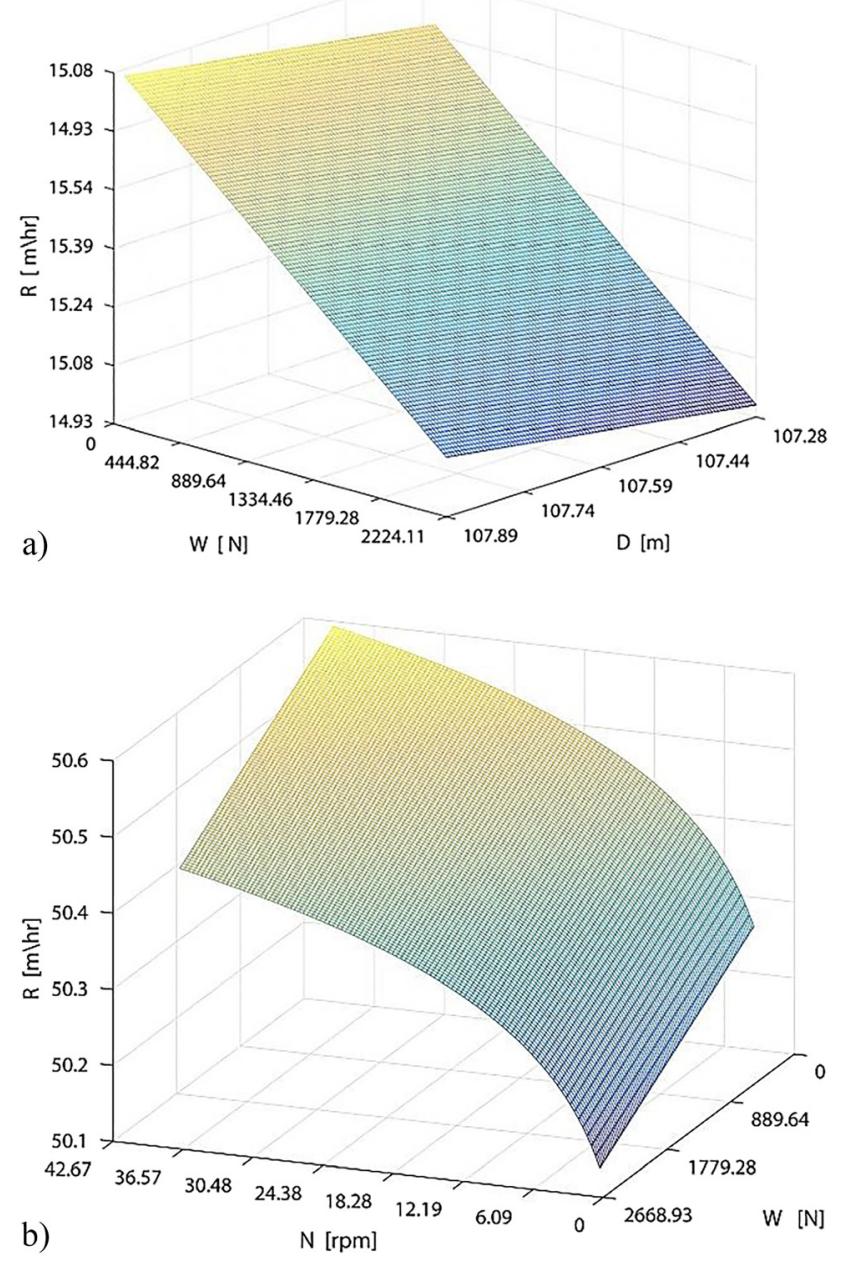

Fig. 6. a) Effect of $W$ and $D$ on ROP; b) effect of $N$ and $W$ on ROP

\section{CONCLUSIONS}

The study was checked and confirmed that the upgrading in the penetration rate was due to optimum weight on bit, rotary speed and other controllable variables. Various metaheuristics algorithms were applied on an actual drilling data from Khangiran field of six wells to find the optimum values for parameters of the Young-Bourgoyne model. High accuracy was achieved between the actual penetration rate and the predicted one. The various metaheuristics algorithms used in this study are capable of accurately simulating large number of drilling variables of a well and reproducing realistic rates of penetration.

\section{REFERENCES}

[1] J. Garcia-Delgado, "Lithology detection in real time", MSc Thesis, Norwegian University of Science and Technology, 2013.

[2] M. Banerjee, P. Chandra, D. Shanker, H. Singh, and V. Singh, "Well function curves for different geometric situations in a largediameter well," Acta Geod. Geophys. Hungarica, vol. 44, no. 4, pp. 439-457, 2009. 
[3] M. J. Kaiser, "A survey of drilling cost and complexity estimation models," Int. J. Pet. Sci. Technol., vol. 1, no. 1, pp. 1-22, 2007.

[4] M. J. Kaiser, "Modeling the time and cost to drill an offshore well," Energy, vol. 34, no. 9, pp. 1097-1112, 2009.

[5] S. Irawan and I. Anwar, "Optimization of weight on bit during drilling operation based on rate of penetration model," J. Aptek, vol. 4, no. 1, pp. 55-64, 2014.

[6] H. A. Hadi and D. A. Al-Obaidi, "Determination of optimum mechanical drilling parameters for an Iraqi field with regression model," in Proceeding of the 2nd International Conference on Iraq Oil Studies, Baghdad, Iraq, Dec. 11-12, 2013, 2013, vol. 11, pp. 25-30.

[7] S. Alsamia, M. S. Mahmood, and A. Akhtarpour, "Estimation of capillary rise in unsaturated gypseous sand soils," Pollack Period., vol. 15, no. 2, pp. 118-129, 2020.

[8] A. T. Bourgoyne, Jr. and F. S. Young, Jr., "A multiple regression approach to optimal drilling and abnormal pressure detection," Soc. Pet. Eng. J., vol. 14, no. 4, pp. 371-384, 1974.

[9] H. Ghafil and K. Jármai, "Comparative study of particle swarm optimization and artificial bee colony algorithms," in Multiscience XXXII. MicroCAD International Multidisciplinary Scientific Conference, Miskolc-Egyetemváros, Hungary, Sep. 5-6, 2018, 2018, pp. 1-6.
[10] H. N. Ghafil and K. Jármai, "Dynamic differential annealed optimization: New metaheuristic optimization algorithm for engineering applications," Appl. Soft Comput., Paper no. 106392, 2020.

[11] H. N. Ghafil and K. Jármai, "Kinematic-based structural optimization of robots," Pollack Period., vol. 14, no. 3, pp. 213-222, 2019.

[12] S. Mirjalili, S. M. Mirjalili, and A. Lewis, "Grey wolf optimizer," Adv. Eng. Softw., vol. 69, pp. 46-61, 2014.

[13] A. A. Heidari, S. Mirjalili, H. Faris, I. Aljarah, M. Mafarja, and H. Chen, "Harris hawks optimization: Algorithm and applications," Futur. Gener. Comput. Syst., vol. 97, pp. 849-872, 2019.

[14] V. Józsa, "Soil classification and determination of over-consolidation from CPTU test in deep excavation," Pollack Period., vol. 8, no. 1, pp. 53-63, 2013.

[15] A. Bahari and A. B. Seyed, "Drilling cost optimization in a hydrocarbon field by combination of comparative and mathematical methods," Pet. Sci., vol. 6, no. 4, pp. 451-463, 2009.

[16] K. Deb, "An efficient constraint handling method for genetic algorithms," Comput. Methods Appl. Mech. Eng., vol. 186, no. 2-4, pp. 311-338, 2000. 